\title{
NORMAL EEG AND ITS DEVELOPMENT IN THE WHITE RAT
}

\author{
NAOSABURO YOSHII AND KAZUO TSUKIYAMA* \\ 2nd Pyhsiological Laboratory, Osaka University Medical School
}

Only a few reports are available concerning the EEG of the rat $(1,2,3,4)$; hence, the normal EEG of adult and infant rats were examined and the reaction to electro-shock to the head of infant rats was also studied.

METHOD

A 4 stage resistance-capacity-coupled amplifier, 0.2 sec. time constant, was used. Its frequency characteristic curve is shown in fig. 1.

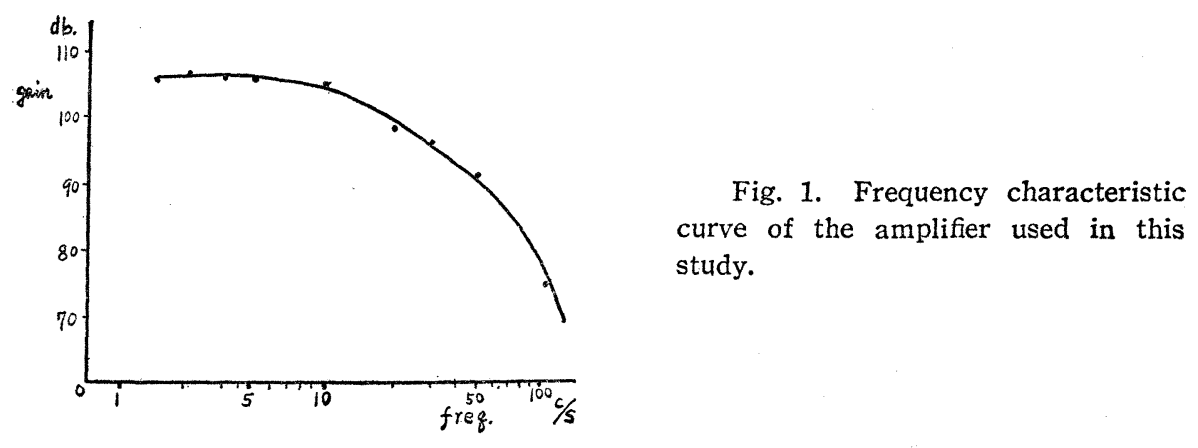

The EEG was recorded by an electromagnetic oscillograph. In each record, average frequency, amplitude and frequency distribution histograms were obtained when necessary, besides observing the wave form. The following three lead-methods were tried and the results were compared.

(1) Skin electrode method: Small solder disk electrodes were attached with electrode-paste to the shaved scalp with collodion.

(2) Drawing pin method: Iron drawing pins were inserted into the skull through the scalp.

(3) Direct method: Small silver disk electrodes were inserted into the skull and fixed on the surface of the dura mater by an operation shown in fig. 2.

In all cases, bipolar leads were employed. The inter-electrode distance was about $1 \mathrm{~cm}$. Records were obtained with the animal in the free state and at rest without anesthesia. In the case of the direct method, experiments were carried out at least 2 or 3 days after operation.

Received for publication April 21, 1951.

* 吉井淔三郎, 筑山一夫 
Fig. 2. Fixation method of lead electrodes (direct method). $A, B:$ Small ( $2 \mathrm{~mm}$. in diameter) silver electrodes (insulated except at the tips). C:Lead wire (insulated). $D$ : Skin. $E$ : Skuli. $F$ : Dura mater. $G$ : Cerebrum.

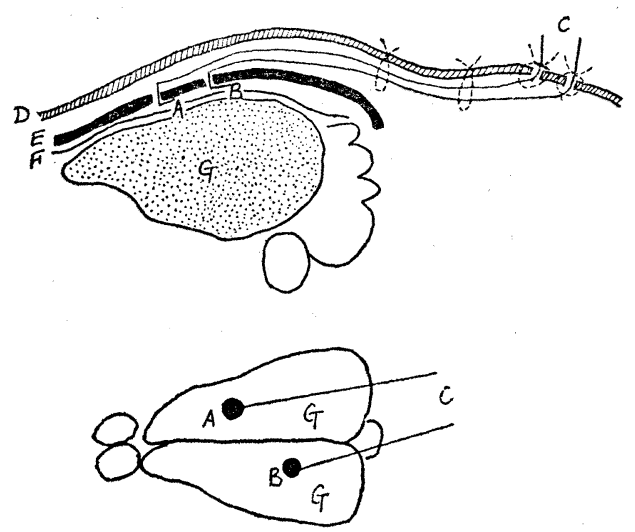

RESULTS

1. Comparison of lead-methods: It was extremely difficult to obtain the correct EEG by means of the skin electrode and drawing pin methods, for the action current of muscles, movements of the electrodes and other artifacts were easily introduced into the electrogram with even the slightest movement of the animal. Good records could sometimes be obtained, nevertheless, when the electrodes were firmly attached and the animal remained quiet (fig. 3, A and $B$ ). Slow waves appeared frequently when the pins were inserted too deep and the brain tissue was injured (fig. $3, C$ ). In the case of the direct leadmethod, almost constant correct records could be obtained without any hindrance if care was taken to fix the electrodes closely to the dura, not to damage the brain tissue and to fix the lead wires closely with the skin as shown in flg. 2. Fig. 3, D, for instance, shows a good record by the direct method which was not influenced by mastication movements. Because of this superiority, the direct method was used in all cases except in the case of infant rats.

Fig. 3. EEG obtained by three lead-methods. $A:$ Skin electrode method (example of good record at rest). $B$ : Drawing pin method (example of good record at rest). $C$ : Drawing pin method (showing predominant slow waves owing to cerebral injury by the electodes, also at rest). $D:$ Direct method (with mastication movements). Upper record: EEG. Lower record: EMG recorded from scalp.

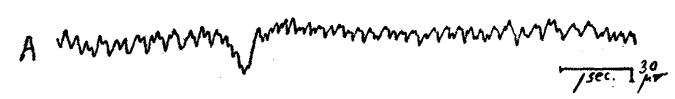

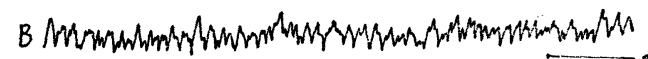
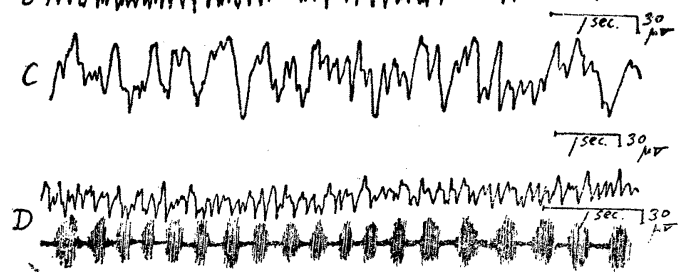

2. Normal EEG of adult rats: Normal EEG of adult rats recorded at rest and in a wakeful state consisted of 7 to 10 (6 to 12) per sec. rhythmic waves (basic waves) and superimposed smaller waves, 16 to 20 or more per sec. Waves slower than 6 per sec. were also observed sometimes but were not predominant. The frequency distribution histogram had two crests, one at the 
basic frequencies and the other at faster frequencies. The average frequency was 9 to 14 per sec. and the average amplitude 30 to $60 \mu \mathrm{V}$ in general. Two. examples are shown in fig. 4.

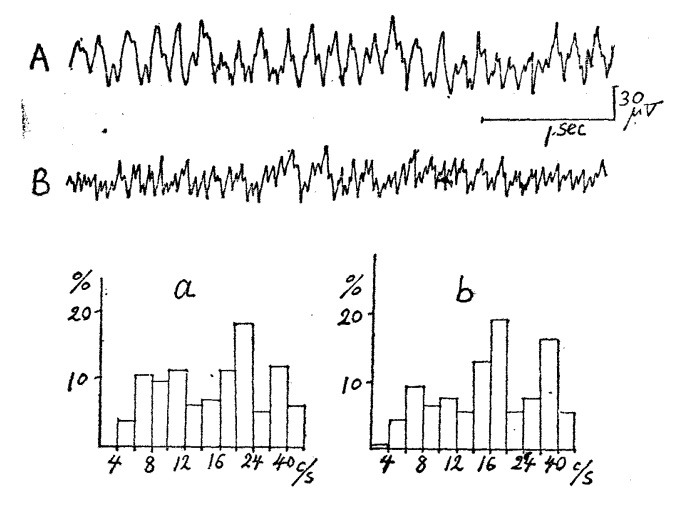

Fig. 4. Normal EEG of two adult rats and their frequency distribution histograms. $A$ and $a:$ A case with predominant basic waves. $B$ and $\boldsymbol{b}:$ Other case with less predominant basic waves.

3. Normal EEG and reaction of infant rats to electro-shock to the head: EEG of infant rats of 4 litter groups were recorded on the 1st or 2nd day after birth until they could be regarded as having almost reached maturity. Use of the skin electrode-method was necessary, since operations were impossible on infant rats. Since the infant animals scarcely moved, no hindrances were introduced into the EEG. Basic waves were observed in the EEG of infant rats even on the 1st or 2nd day after birth, but their regularity was less marked and the amplitudes were smaller than those in adults. It required about 3 weeks after birth for the EEG to attain those of adults. An example of an electroencephalographic development is shown in fig. 5 .

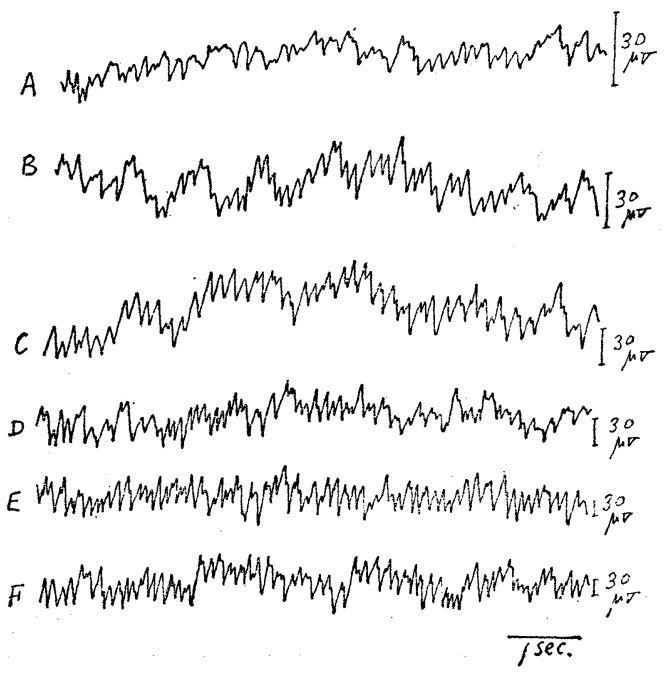

Fig. 5. EEG development of infant rats of a litter (skin electrodes). $A$ : 2nd day, $B: 5$ th day, $C: 10$ th day, $D:$ 14th day, $E: 21$ st day after birth. $F$ : Adult rat.

Electro-shocks were given to the head of infant rats at various periods after birth. $60 \mathrm{c} / \mathrm{s}$. alternate current was transmitted 1 second at intensities of 
$10,20, \ldots, 100 \mathrm{~V}$ through both aural orifices by pincer form brass electrodes of Shimizu (5). Until the 3rd or the 4th day after birth, convulsions did not occur and no change was seen in EEG by electro-shock even with the strongest voltage (fig. $6, A$ and $B$ ). On the 4th or 5th day, the EEG showed high voltage convulsive patterns but actual convulsive movements of the limbs were still not observed (fig. $6 C, D$ and $E$ ). It was after about 7 days that convulsion of the limbs occurred associated with EEG changes.

Fig. 6. EEG of infant rats by electro-shock. $A$ : 1st day, normal record. $B$ : same, immediately after shock $(20 \mathrm{~V}$, 1 sec.), no convulsion, EEG unchanged. $C: 5$ th day, normal record. $D$ : same, immediately after shock $(20 \mathrm{~V}, 1$ sec. $)$, no convulsion but convulsive activity in EEG. $E$ : same, 4 minutes after shock.

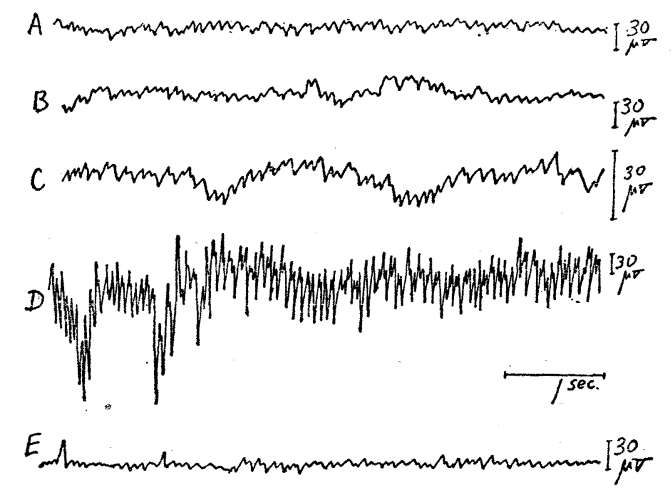

DISCUSSION

Travis and Dorsey (1) observed very fast waves in the EEG of rats, but the amplifier they used was of such a high frequency and 100-800 c/s. ranges were chiefly dealt with. It is impossible, therefore, to compare their results with ours. Lindsley et al. (2) recorded the EEG of rat in audiogenic seizure, but they did not mention the normal pattern in detail. But about 8 per sec. basic waves can be noticed in their control records. Overholser et al. (3) reported the EEG of young rats, 4-8th weeks after birth, and showed that the average frequency was 30.8 per sec. This is much higher than that seen in our experiment. Since the results mentioned by the above investigators were all obtained from skin electrodes, it is difficult to regard them as strictly correct. Katsura (4) recorded the EEG of rats by means of intracranially inserted silver electrodes and observed 6.2 to 11 per sec. basic waves and 12 to 16 per sec. as the average frequency. These results are closer to ours.

It is difficult to obtain accurate EEG of infant rats, since the direct method cannot be used and the animals are quickly weakened during the process of recording. Nevertheless, it may be said that basic waves, though more irregular and smaller in voltage than those of adults, are already present in the new born rat and that it takes about 3 weeks after birth for normal adult patterns to appear. Sometimes, especially in the early period after birth, no basic waves were observed and irregular slow waves were predominant. This abnormality is regarded as the result of the weakened state of the infant rat during the expeimental procedure.

It is an interesting finding that convulsive activity is observed in EEG without motor manifestation by electro-shock on the 4 th or 5 th day after birth and 
that the actual convulsive movements are observed later at about the 7th day. Hiraiwa (6) in our laboratory investigated the development of the flexion reflex on infant rats and observed that the reflex latency was shortened abruptly on the 7 th day and the isometric flexion reflex record became almost the same as that of the normal adult on about the 20-25 days after birth. Comparing this with our results, it may be said that the period when reflex latency is abruptly shortened coincides fairly well with the period when actual convulsion is induced by electro-shock, and further, when the isotonic reflex-curve becomes almost the same as in the adult, the EEG also shows an almost normal adult pattern. These results present a powerful suggestion to the problem of development of the central nervous system in the white rat. It has already been shown in cats and dogs that we cannot induce movements of the limbs by faradic stimulation on the motor cortex until about 10 days after birth and that epileptic convulsions cannot be induced until about 2 months after birth (7).

\section{SUMMARY}

1. Three lead-methods for recording the EEG of rats were compared and it was found that the direct method gives the most accurate results.

2. Normal EEG of the adult rat is characterized by rhythmical 7-10 (6-12) per sec. basic waves and superimposed smaller fast waves. Alteration of proportion of these two components gave individual differences of normal records. Slow waves of less than 6 per sec. were also present but were not significant.

3. EEG of the new born rat showed basic waves, though irregular and small, and it was about 3 weeks after birth that the EEG became almost identical with that of normal adult rats.

4. Until the 3rd or the 4th day after birth, no changes in EEG or movement were noted following electro-shock to the head. On the 4 th or 5th day, a convulsive EEG pattern was noticed but it was about 7 days that the actual convulsion of limbs was associated with EEG changes.

\section{REFERENCES}

1. TRAvis, L. E. AND J. M. DORSEY. Arch. Neurol. Psychiat. 28 : 331, 1932.

2. LINDSLEY, D. B., F. W. FINGER AND C. E. HENRY. J. Neurophysiol. 5 : 185, 1942.

3. Overholser, M. D., J. R. Whitley, B. L. O'Dell AND A. G. Hogan. Science 111: $65,1950$.

4. KATSURA, J. Brain Reserches 4: 1, 1949.

5. Shimizu, S. J. phsiol. Soc. Jap. 10: 308, 1948.

6. HIRAIWA, K. unpublished.

7. FEldman, W. M. The Principles of Ante-Natal and Post-Natal Child Physiology 219220, 1920 (London). 